\section{Defizite in der Rehabilitation}

\section{Deutsche Kardiologen genießen zwar den Ruf des Interventions-Weltmeis- ters. Was die Rehabilitation ihrer Herz-Kreislauf-Patienten betrifft, sind sie al- lerdings von einer Spitzenposition weit entfernt. Das zeigt zumindest das europäische EUROASPIRE-III-Register zur Verordnung von Rehabilitation.}

$\mathrm{D}$ as EUROASPIRE-III-Register stützt sich auf 76 Zentren in 22 europäischen Ländern. 13.935 unter 80-jährige Patienten nach kardiovaskulärem Ereignis oder Revaskularisation wurden aufgenommen, 8.845 (77\%) konnten interviewt werden. Nur knapp $56 \%$ der KHKPatienten wurde im Rahmen einer Stichprobe eine Reha empfohlen, wobei über 91\% dieses Angebot gerne annahmen.

Vom Standpunkt der Reha-Mediziner ist dieser statistische Schnappschuss ein Beweis für die ignorante Haltung vieler Kliniker gegenüber der Reha. Dr. Gesine Dörr, Angermünde (Uckermark) wies darauf hin, dass es sich in diesem Register vornehmlich um kardiologische $\mathrm{Pa}$ tienten handelt. „Wie schlecht sind dann erst unsere PAVK-Patienten versorgt?", fragte sie bitter. Zunächst fällt Dörr die niedrige Motivation der Patienten nach peripherer Revaskulisation auf.

\section{Frauen sind schlechter versorgt}

Erschütternd sei besonders die schlechte Versorgung der Frauen mit PAVK. In der Statistik der Rehabilitationsindikatoren von 2013 steht bei den Männern die KHK vor den Rückenschmerzen und dem Schlaganfall auf den ersten Plätzen. Bei Frauen fällt die KHK auf Platz 14, die Herzinsuffizienz taucht unter den ersten 20 Indikatoren gar nicht auf und die PAVK wird gar nicht mehr erfasst.

Tatsächlich schauen die Reha-Angiologen etwas neidisch auf ihre kardiologischen Partner, die es schon vor Jahrzehnten geschafft haben, ihre Infarktpatienten in Koronargruppen zu organisieren und damit zumindest bei einem Teil

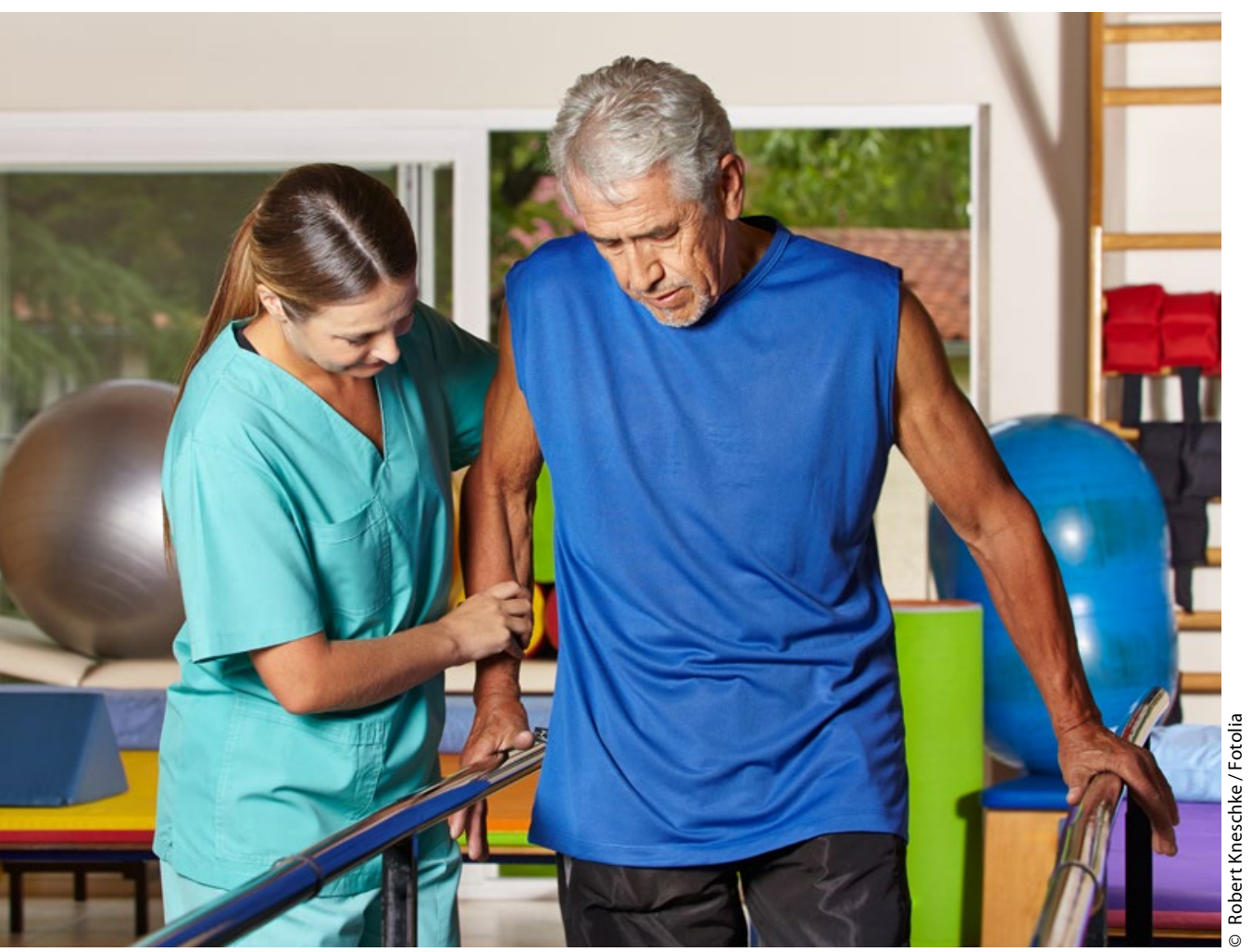

In den Genuss eines Gehtrainings kommen leider nur wenige PAVK-Patienten.

\section{PAVK in Deutschland: Stationäre Perspektiven}

- Die Patientenzahl bei PAVK ist von 2005 bis 2009 um $20 \%$ angestiegen. Die stationäre Mortalität liegt unverändert hoch bei $8,4 \%$. Das mittlere Patientenalter beträgt ca. 72 Jahre. - Der Anteil der Diabetiker ist leicht angestiegen von 32,5 auf $37,4 \%$.

_ Frauen sind seltener betroffen als Männer: 34,5\%.

— Nur bei $50 \%$ der CLI-Patienten erfolgte eine arterielle Gefäßrekonstruktion.

_ Die endovaskuläre Therapie wird zunehmend häufiger eingesetzt und erreicht ein Anteil von 19,7\%, dagegen ist die periphere Bypasschirurgie rückläufig (11,2\%).

Quelle: Nach Malyar N. et al. European Heart J 2013; 34: 2706-14

der Patienten wesentliche Reha-Ziele erreicht haben. Für die PAVK wären Gefäßsportgruppen dringend erforderlich, bislang gibt es aber fast nur Wunschvorstellungen. Die Umsetzung scheitert oftmals an hohen Finanzierungshürden.

Dabei ist die körperliche Aktivität samt Gehtraining bei Claudicatio intermittens (CI) nach der aktuellen europäischen Leitlinie die konservative Therapie der Wahl. Einen genaueren Blick auf die Versorgungsrealität in Sachen Gehtraining hat eine Gruppe des Universitäts-Herzzentrums Freiburg-Bad Krozingen geworfen. Von zehn deutschen Zentren wollte man wissen, ob bei den Patienten mit PAVK Stadium II Gehtraining erfolgte, und zwar vor oder anstatt oder nach einer Intervention bzw.Operation. Knapp 1.300 Fragebögen wurden verschickte, der Rücklauf lag bei $49 \%$. 408 Bögen waren schließlich auswertbar. Die wesentlichen Ergebnisse:

_Nur 16,2\% der Cl-Patienten haben im Rahmen der Therapie leitliniengerecht Gefäßsport betrieben.

- Viele Patienten berichten, die vorgesehene Ersttherapie war interventionell oder operativ.

- Von den Gefäßsport treibenden CIPatienten sahen $70 \%$ die Behandlung als erfolgreich an.

Dr. med. Jochen Aumiller 